\title{
Power generation by reverse electrodialysis in a single-layer nanoporous membrane made from core-rim polycyclic aromatic hydrocarbons
}

\author{
Xue Liu ${ }^{1}$ ', Meng He', Dario Calvani ${ }^{\circledR}$, Haoyuan Qi ${ }^{2}{ }^{2}$, Karthick B. Sai Sankar Gupta1, \\ Huub J. M. de Groot ${ }^{1}$, G. J. Agur Sevink', Francesco Buda', Ute Kaiser ${ }^{\left(\mathbb{D}^{2}\right.}$ and Grégory F. Schneider ${ }^{1{ }^{1 凶}}$
}

Nanoporous graphene and related atomically thin layered materials are promising candidates in reverse electrodialysis research owing to their remarkable ionic conductivity and high permselectivity. The synthesis of atomically thin nanoporous membranes with a narrow pore size distribution, however, remains challenging. Here, we report the fabrication of nanoporous carbon membranes via the thermal crosslinking of core-rim structured monomers, that is, polycyclic aromatic hydrocarbons. The mechanically robust, centimetre-sized membrane has a pore size of $3.6 \pm 1.8 \mathrm{~nm}$ and a thickness of $2.0 \pm 0.5 \mathrm{~nm}$. When applied to reverse electrodialysis, the nanoporous carbon membrane offers a high short-circuit current with an output power density of $67 \mathrm{~W} \mathrm{~m}^{-2}$, which is about two orders of magnitude beyond that of the classic ion-exchange membranes and current prototype nanoporous membranes reported in the literature. Crosslinked and atomically thin porous polycyclic aromatic hydrocarbon membranes therefore represent new scaffolds that will revolutionize the rapidly developing fields of sustainable energy and membrane technology.

W ith reverse electrodialysis, electricity is generated directly from mixing fresh water with salty water ${ }^{1}$. The technology is not only suitable to generate electricity for industrial and domestic utilization but also shows a bright future to power implant materials, for example, pacemakers and prosthetic devices ${ }^{2,3}$. The ion-exchange membrane-the most crucial component-separates fresh water from salty water to avoid the Gibbs free energy loss resulting from direct mixing ${ }^{4}$. Therefore, considerable efforts have been devoted to the development of high-performance ion-exchange membranes with high permselectivity (the ratio of the permeabilities of ions) and ionic conductivity (the tendency of an ion to pass through the membrane) $)^{5,6}$.

The micrometre-thick polymer-based membranes used nowadays show decent ion permselectivity, with, however, limited ionic conductivity 7,8 . The ion conductivity scales inversely with the membrane thickness and, therefore, can be circumvented by reducing the membrane thickness down to the atomic level ${ }^{9}$. Besides, theoretical and experimental works have demonstrated that atomically thin membranes with nanometre pores exhibit ultra-permeability as well as high selectivity ${ }^{10-13}$, making them promising candidates for reverse electrodialysis as well as water desalination and fuel cells.

In practice, nanopores can be introduced into two-dimensional (2D) materials by physical methods such as electron-beam irradiation, plasma etching and ion bombardment, with the drawback that not only the chemical functionality of the pores is poorly controlled, but also the scalability of the process remains elusive'. Although the bottom-up fabrication of 2D nanoporous membranes can solve these problems, it remains technically challenging because of the lack of reliable methods to produce high-quality large-scale $2 \mathrm{D}$ membranes with nanopores ${ }^{14,15}$. Two-dimensional metal-organic frameworks and covalent organic frameworks with well-defined nanopores were also explored, with the main drawbacks that the chemical and mechanical stability in solutions limits the application of such ion-selective membranes ${ }^{16,17}$.

Here, we demonstrate the bottom-up synthesis of molecularlythin carbon membranes with an intrinsic, tight size distribution of nanometre pores from core-rim structured monomers via a twostep approach. Importantly, we show that the membrane exhibits excellent ionic conductivity and outstanding output power density, which is directly suitable for nanofluidics, reverse electrodialysis, membrane separation and nanopore applications.

\section{Membrane fabrication}

In this work, centimetre-sized molecularly-thin nanoporous carbon membranes with nanometre pores are fabricated in two steps (Fig. 1a). In the first step, a core-rim structure polycyclic aromatic hydrocarbon monomer hexa(2,2'-dipyridylamino)hexabenzocoronene (HPAHBC) (the synthesis and characterization details are available in the Methods), consisting of six flexible dipyridylamino groups as the rim and one hexabenzocoronene (HBC) rigid core (Fig. 1b), is spread from a chloroform solution at the air/water interface in a Langmuir-Blodgett trough. The flexible dipyridylamino rims enhance the solubility of the $\mathrm{HBC}$ core in chloroform. The amphiphilic nature of HPAHBC (dipyridylamino rim is hydrophilic, $\mathrm{HBC}$ core is hydrophobic) allows forming a monolayer of HPAHBC on the water surface. Subsequently, the monomers are compressed to specific surface pressures $\left(3 \mathrm{mN} \mathrm{m}^{-1}, 10 \mathrm{mN} \mathrm{m}^{-1}, 20 \mathrm{mN} \mathrm{m}^{-1}\right.$ and $30 \mathrm{mN} \mathrm{m}^{-1}$ ) (Fig. 1c). The mean molecule area (MMA) of $59 \AA^{2}$-determined by extrapolating the compression isotherm to a zero surface pressure ${ }^{18}$ - is too small for HPAHBC lying flat on the water surface, suggesting that HPAHBC adopts an edge-on orientation (Supplementary Fig. 1).

'Leiden Institute of Chemistry, Faculty of Science, Leiden University, Leiden, The Netherlands. ${ }^{2}$ Central Facility of Electron Microscopy, Electron Microscopy Group of Materials Science, Ulm University, Ulm, Germany. $\varpi_{e}$-mail: g.f.schneider@chem.leidenuniv.nl 

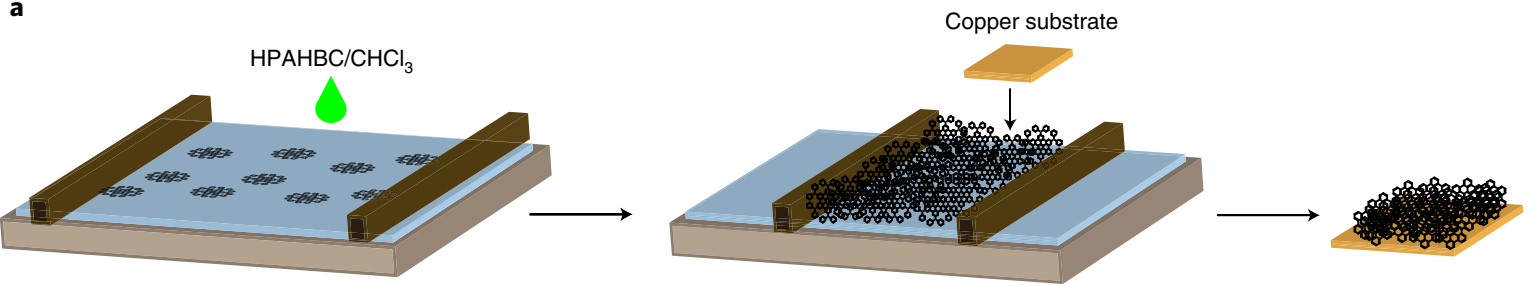

b

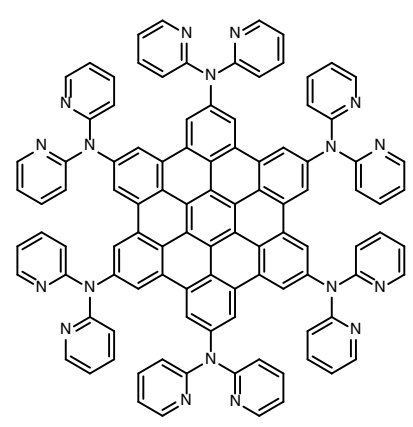

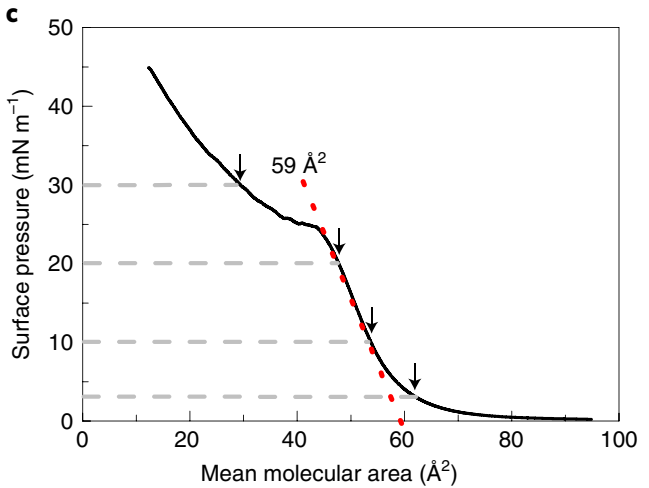

d
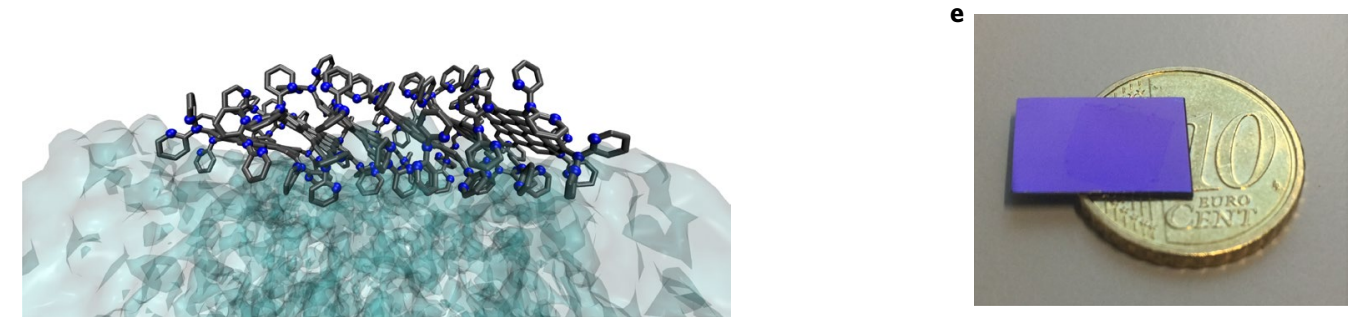

Fig. 1 | The preparation of atomically thin nanoporous carbon membranes. a, In a Langmuir-Blodgett trough, a $1 \mathrm{mg} \mathrm{ml}^{-1}$ solution of $\mathrm{HPAHBC}$ in chloroform is deposited dropwise at the air/water interface and compressed to 3,10,20 and $30 \mathrm{mN} \mathrm{m}^{-1}$. After compression, the copper foil is brought into contact with the compressed HPAHBC layer using the Langmuir-Schäfer approach, resulting in the transfer of the HPAHBC monolayer onto the copper foil. Then, the HPAHBC monolayer is annealed at $550^{\circ} \mathrm{C}$ under vacuum ( $1 \mathrm{mbar}$, argon atmosphere) for 20 min. $\mathbf{b}$, Chemical structure of the $\mathrm{HPAHBC}$ monomer. c, Compression isotherm of HPAHBC spread at the air/water interface at $24^{\circ} \mathrm{C}$ measured in a Langmuir-Blodgett trough. The four surface pressures used for the Langmuir-Schäfer HPAHBC layer transfer are indicated with grey dashed lines. The MMA is determined to be $59 \AA^{2}$ by extrapolation of the isotherm (red dotted line). d, A representative snapshot from the MD simulation including six HPAHBC molecules on the water surface showing the most frequent orientation and packing adopted by the molecules. $\mathbf{e}$, A digital photograph of a crosslinked membrane transferred onto a $\mathrm{Si} / \mathrm{SiO}_{2}$ wafer after annealing. The size of the membrane is about $1 \times 1 \mathrm{~cm}^{2}$.

The orientation of the HPAHBC molecules on the water surface has been further explored by molecular dynamics (MD) simulations. A water slab has been considered with two interfaces with an increasing number of HPAHBC molecules on the water surfaces (see section 'Simulations' in the Methods for more information). The HPAHBC molecules experience two competing intermolecular interactions that drive the arrangement of the HPAHBC molecules on the water surface: (1) the hydrogen bond interaction between the dipyridylamino rim and the water molecules and (2) the $\pi-\pi$ stacking interaction between the aromatic HBC cores of the molecules. A single HPAHBC molecule isolated on the water surface tends to maximize the number of hydrogen bonds (on average six), with the $\mathrm{HBC}$ core essentially parallel (tilt angle $\sim 10^{\circ}$ ) to the water surface (Supplementary Fig. 2 and Extended Data Fig. 1). However, with an increasing number of interacting HPAHBC molecules on the water surface, the nonlinear cooperative $\pi-\pi$ stacking interaction $^{19}$ soon becomes the dominant contribution in determining the overall arrangement (Supplementary Table 1): the molecules adopt a parallel-displaced stacking with the $\mathrm{HBC}$ core plane forming a tilt angle of around $\sim 35^{\circ}$ with the water surface (Extended Data Fig. 1d,e). This is clearly visible in Fig. 1d, which shows the representative snapshot from the MD simulation including six HPAHBC molecules. In this arrangement, each molecule forms on average two hydrogen bonds (Extended Data Fig. 1f) and gives an MMA around $100 \AA^{2}$. From the trend observed in the MD simulations (Extended Data Fig. 1e) we expect that the tilt angle will approach a vertical orientation $\left(90^{\circ}\right)$ by increasing the concentration of molecules on the surface. This would also result in a lower MMA, approaching the experimentally determined value.

In the second step, the HPAHBC monolayer is transferred using the Langmuir-Schäfer method onto a copper substrate and annealed under vacuum ( $1 \mathrm{mbar}$, argon atmosphere) at $550^{\circ} \mathrm{C}$, yielding a crosslinked membrane. The dipyridylamino rim is decomposed upon annealing while the $\mathrm{HBC}$ core is thermally stable. The large molecular weight $(1,537 \mathrm{Da})$ of HPAHBC is crucial to prevent sublimation of the molecule upon annealing ${ }^{15}$. For further characterizations, the membrane is transferred onto a $\mathrm{Si} / \mathrm{SiO}_{2}$ wafer (Fig. 1e and Supplementary Fig. 3) and onto holey supports using the so-called 'wedging transfer method' typically used for graphene ${ }^{20}$.

\section{Structural characterization}

The membrane was then fully characterized by Fourier-transform infrared spectroscopy (FTIR), X-ray photoelectron spectroscopy (XPS), Raman spectroscopy, atomic force microscopy (AFM), 


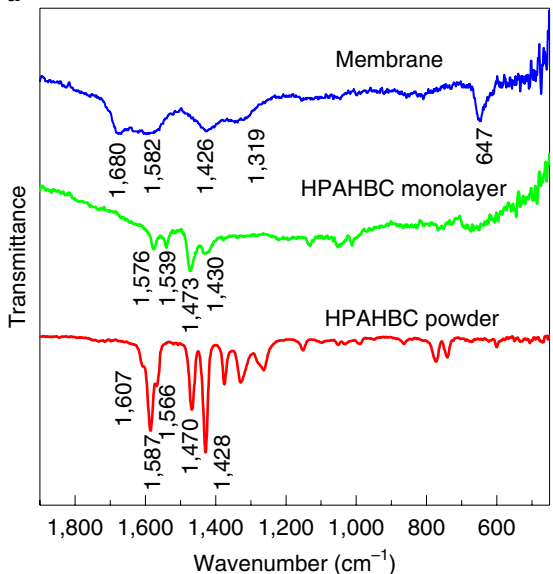

d

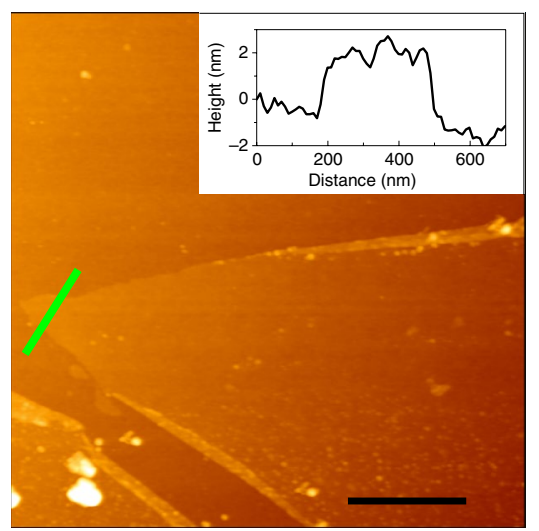

b
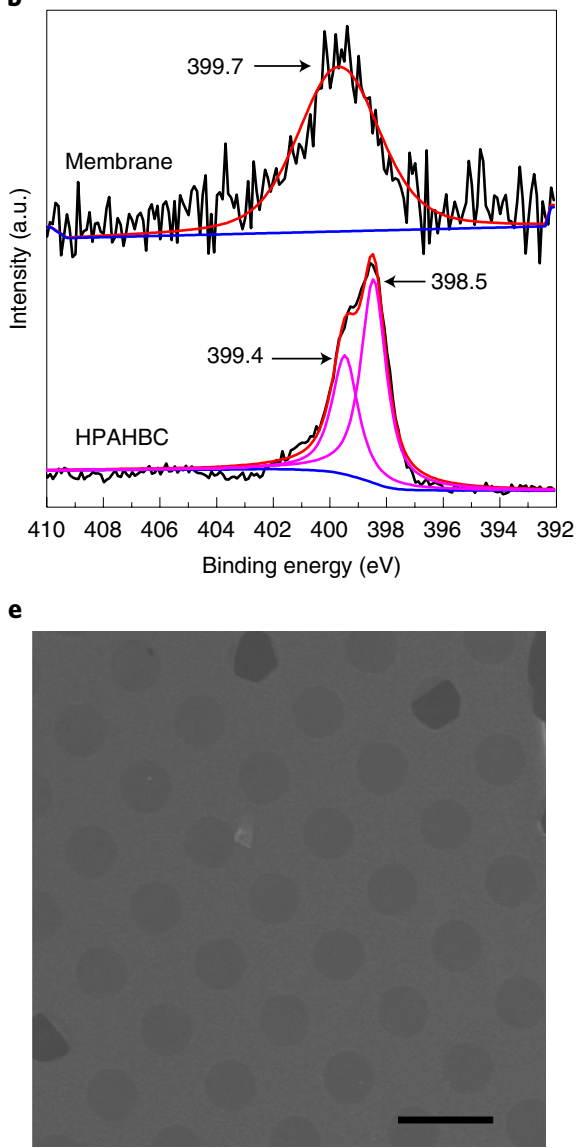

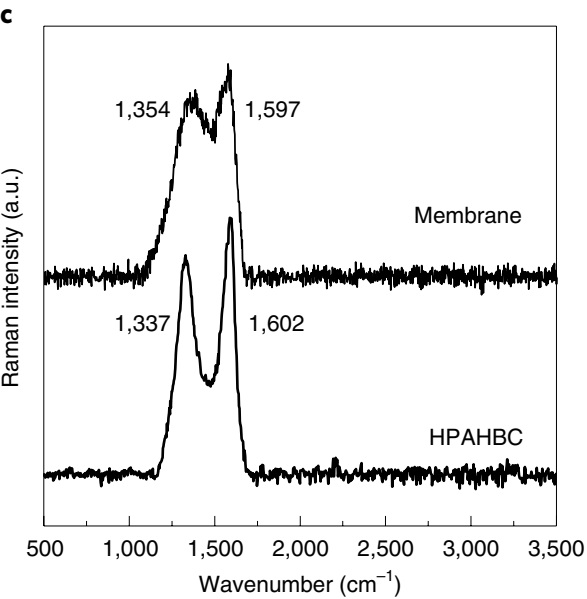

$\mathbf{f}$

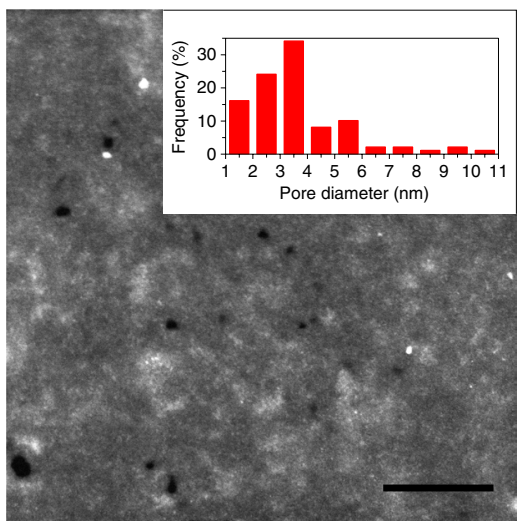

Fig. 2 | Characterization of the membrane prepared at $10 \mathrm{mN} \mathrm{m}^{-1}$. a, FTIR spectra of HPAHBC powder (red), HPAHBC monolayer transferred onto a copper substrate before annealing (green) and after thermal annealing (blue). The characteristic FTIR peaks of the pyridyl group after annealing are not observed, indicating the decomposition of the pyridyl groups. b. High-resolution N 1s XPS spectra of HPAHBC monomer and the membrane. The pyridinic N with a binding energy of $398.5 \mathrm{eV}$ is not detectable in the membrane, confirming the decomposition of the pyridyl groups. Pink curve, Gaussian components of the XPS spectra; red curve, sum curve of Gaussian components; blue curve, baseline. c, Raman spectra of HPAHBC powder and the membrane showing the characteristic $D$ and $G$ peaks around 1,350 and 1,600 $\mathrm{cm}^{-1}$ under the excitation of a green laser at $532 \mathrm{~nm}$, indicating the existence of polycyclic aromatic hydrocarbons ( $\mathrm{HBC}$ ). d, AFM image of the membrane on a $\mathrm{Si} / \mathrm{SiO}_{2}$ wafer. Inset: height profile of the cross-section at the location highlighted by the solid green line in the AFM scan, the thickness of the film is about $2 \pm 0.5 \mathrm{~nm}$. Scale bar, $1 \mu \mathrm{m}$. e, SEM image of the membrane on a gold QUANTIFOIL grid perforated with an array of $2 \mu \mathrm{m}$ diameter holes. Scale bar, $4 \mu \mathrm{m}$. $\mathbf{f}$, HAADF-STEM image of the membrane. The nanopores appear dark on a bright background. Scale bar, $40 \mathrm{~nm}$. Inset: the relative frequency of pore diameters (nm) derived from 100 pores in 10 images (Fig. $2 f$ and Supplementary Fig. 9 ).

scanning electron microscopy (SEM), high-resolution transmission electron microscopy (HRTEM) and high-angle annular darkfield scanning transmission electron microscopy (HAADF-STEM). The FTIR spectrum of the HPAHBC powder shows strong bands at $1,587,1,470$ and $1,428 \mathrm{~cm}^{-1}$, which can be assigned to the pyridyl groups with $\mathrm{C}-\mathrm{N}$ and $\mathrm{C}-\mathrm{C}$ vibrations, $\mathrm{H}-\mathrm{C}-\mathrm{N}$ bending vibrations and $\mathrm{H}-\mathrm{C}-\mathrm{C}$ bending vibrations (Fig. 2a) ${ }^{21}$. The positions and relative intensity of those peaks in the HPAHBC monolayer are different from the HPAHBC powder in the FTIR spectrum, further confirming the edge-on orientation of HPAHBC in the monolayer ${ }^{22}$. After thermal annealing at $550^{\circ} \mathrm{C}$, the typical peaks for the pyridyl groups disappear, indicating the complete decomposition of the pyridyl groups. Only five broad bands are observed, which are typical for polycyclic aromatic hydrocarbons, confirming that the HBC cores remain, which is not surprising since HBC is thermally stable up to $800^{\circ} \mathrm{C}$ (ref. ${ }^{23}$ ).

The decomposition of the pyridyl groups was further confirmed by the XPS measurements (Fig. 2b). The $\mathrm{N} 1 s$ binding energy of the HPAHBC monomer can be fitted to two peaks at 398.5 and $399.4 \mathrm{eV}$, corresponding to the pyridinic nitrogen and tertiary nitrogen $^{24,25}$. The $\mathrm{N} 1 s$ binding energy of the membrane contains only one peak at $399.7 \mathrm{eV}$ that can be ascribed to the tertiary nitrogen, demonstrating the decomposition of the pyridyl groups ${ }^{26}$. Further, the measured $\mathrm{N} / \mathrm{C}$ atomic ratio of HPAHBC is 1:5.7, which is higher than the ratio measured for the membrane (1:38.1), indicating the loss of nitrogen atoms and the decomposition of the pyridyl groups during annealing. The $\mathrm{C} 1 s$ binding energy of the membrane contains three peaks, which can be ascribed to $s p^{2}$ carbon, C-N/C-O and $\mathrm{C}=\mathrm{O}$ (Supplementary Fig. 4) ${ }^{27}$. The decomposition of the pyridyl groups may result in the interaction between active carbon species with oxygen, which is likely to be oxygen atoms adsorbed on the sample surface ${ }^{28}$.

As illustrated in the Raman spectra (Fig. 2c), HPAHBC shows strong $\mathrm{G}$ and $\mathrm{D}$ bands located at $1,602 \mathrm{~cm}^{-1}$ and $1,337 \mathrm{~cm}^{-1}$, arising from the bond stretching of $s p^{2}$-bonded pairs and of $s p^{3}$-hybridized defects, respectively ${ }^{29}$. The characterized Raman peaks of pyridine (around 1,010 and $1,030 \mathrm{~cm}^{-1}$ ) could not, however, be observed due to their low signal-to-noise ratio in this spectral region ${ }^{30}$. Similarly to the HPAHBC monomer, the spectrum of the membrane exhibits a strong $\mathrm{G}$ peak at $1,597 \mathrm{~cm}^{-1}$ as well as a D peak at $1,354 \mathrm{~cm}^{-1}$. The typical 2D peak for graphene and graphene nanoribbons is not observed, indicating that the $\mathrm{HBC}$ cores are still isolated from each 


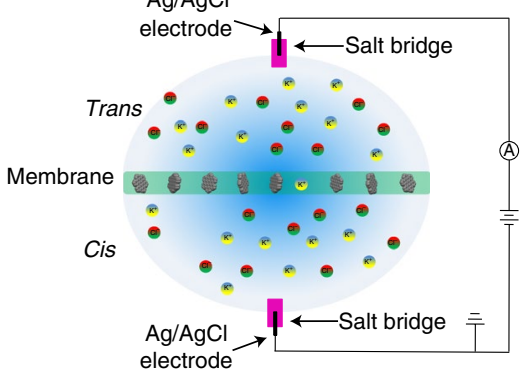

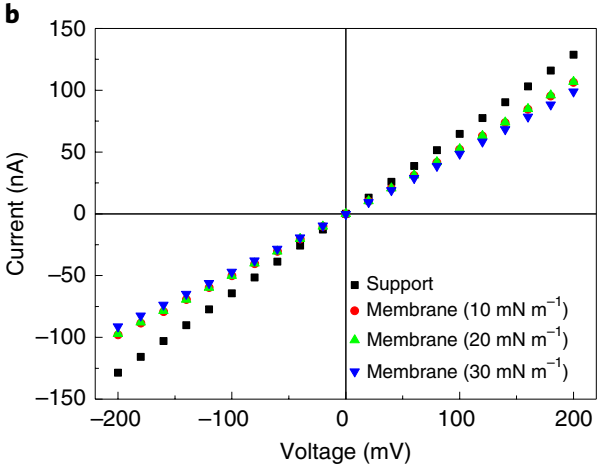

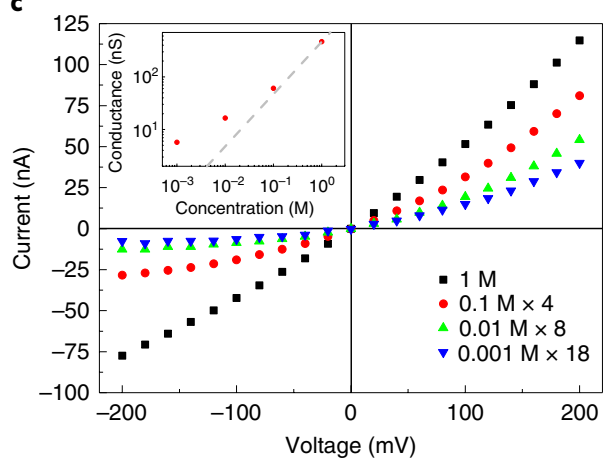

Fig. $3 \mid$ Pore density and transmembrane ionic current measurements. a, The experimental set-up. The membrane is freestanding over a $1 \mu \mathrm{m}$ diameter glass aperture with the same concentration of $\mathrm{KCl}$ on both sides of the membrane. The ions are driven through the pores of the membrane by applying an electrical potential. $\mathbf{b}$, The $I-V$ characteristics of the membranes prepared at different surface pressures $\left(10 \mathrm{mN} \mathrm{m}^{-1}, 20 \mathrm{mN} \mathrm{m}^{-1}\right.$ and $\left.30 \mathrm{mN} \mathrm{m}^{-1}\right)$ on a glass support in $1 \mathrm{M} \mathrm{KCl}$ solution at $\mathrm{pH}$ 3. c, The $I-\mathrm{V}$ characteristics of the membrane prepared at $10 \mathrm{mN} \mathrm{m}^{-1}$ suspended on a glass pore for a $\mathrm{KCl}$ solution at $\mathrm{pH} 7$. For clarity, the current for concentrations below $1 \mathrm{M}$ is magnified by the factors given in the legend. Inset: the conductance for the membrane under small bias. The experimental conductance of the membrane decreases nonlinearly as the $\mathrm{KCl}$ concentration decreases, indicating the existence of surface charge. The grey dashed line is a guide showing the linear decrease of the ionic conductance with decreasing salt concentration.

other in the crosslinked film ${ }^{31-33}$. The Raman spectra of the membrane obtained at higher surface pressure exhibit narrower D and G peaks (Supplementary Fig. 5).

The AFM analysis reveals the uniform flat morphology of the membrane with a thickness of around $2 \pm 0.5 \mathrm{~nm}$ and a root-meansquare roughness $\left(R_{\mathrm{rms}}\right)$ of $1.1 \mathrm{~nm}$ (Fig. $2 \mathrm{~d}$ ). The membranes prepared at different surface pressures show similar morphologies and thicknesses (Supplementary Fig. 6). The SEM and HRTEM images confirm that the membrane is smooth and uniformly flat (Supplementary Figs. 7 and 8). The membranes prepared above $10 \mathrm{mN} \mathrm{m}^{-1}$ are robust enough to be freestanding over open apertures as large as $2 \mu \mathrm{m}$ in diameter with only a few cracks (Fig. $2 \mathrm{e}$ and Supplementary Fig. 7). The HAADF-STEM images reveal the presence of nanopores with a narrow size distribution (Fig. 2f). The average pore diameter is $3.6 \mathrm{~nm}$ with a standard deviation of $1.8 \mathrm{~nm}$ derived by analysing 100 pores in 10 images (Fig. $2 \mathrm{f}$ and Supplementary Fig. 9).

To study the annealing process, we also performed solid-state nuclear magnetic resonance (NMR) of the HPAHBC powder. The chemical shifts of the solid-state HPAHBC sample are comparable to those for the molecule in solution, indicating a weak $\pi-\pi$ interaction between HPAHBC molecules in the solid state (Supplementary Fig. 10). Additionally, ${ }^{13} \mathrm{C}$ cross-polarization magic-angle spinning (CPMAS) experiments were performed on the samples treated at high temperatures (Supplementary Fig. 11 and Extended Data Fig. 2). By increasing the temperature, the carbon peaks that are assigned to the dipyridylamino rim (marked with blue dots) vanished slowly, indicating the decomposition of the dipyridylamino rim. The ${ }^{13} \mathrm{C}$ signals for the $\mathrm{HBC}$ core (marked with red dots) broaden when the sample is heated, revealing the amorphous nature of the membrane ${ }^{34,35}$. An anisotropy change is observed between the data collected from samples annealed at $550^{\circ} \mathrm{C}$ and without annealing, indicating chemical structure changes during the annealing process. The chemical shifts for the molecules in solution and the solid state are listed in Supplementary Table 2, which also shows the theoretical predictions. The root mean square deviation (RMSD) between theory and experimental results is $\sim 7 \mathrm{ppm}$.

\section{Pore density characterization}

The characterization of the membrane structure reveals that the membrane is porous. To quantify the pore density of the membranes, we studied the transport properties of hydrated ions, $\mathrm{K}^{+}$and $\mathrm{Cl}^{-}$, in a typical cis/trans nanopore set-up (Fig. $3 \mathrm{a}$ ). $\mathrm{KCl}$ is used because the
$\mathrm{K}^{+}$and $\mathrm{Cl}^{-}$show similar bulk mobility and negligible liquid junction potential ${ }^{36}$. For this purpose, the membranes are transferred on top of a glass disc perforated with a $1-\mu \mathrm{m}$-wide through-hole and immersed on both sides with an aqueous solution containing $1 \mathrm{M}$ of $\mathrm{KCl}$ at $\mathrm{pH} 3$ to avoid the influence of the membrane surface charge on transmembrane ionic transport. The current-voltage $(I-V)$ characteristics of the membranes show slight nonlinearity over the studied voltage range (Fig. 3b). The pore density of the membrane can be calculated by using equation $(1)^{37}$

$$
G_{\text {membrane }}=n G_{\text {individual }}=n \sigma\left(\frac{4 l}{\pi D^{2}}+\frac{1}{D}\right)^{-1}
$$

where $G_{\text {membrane }}$ is the conductance of the whole nanopore array, $n$ is the number of nanopores in the freestanding membrane area, $G_{\text {individual }}$ is the conductance of each pore, $\sigma$ is the conductivity of the solution $\left(10.5 \mathrm{~S} \mathrm{~m}^{-1}\right), l$ is the length of the pore $(2 \mathrm{~nm}$, the thickness of the membrane determined by AFM), and $D$ is the average nanopore diameter $(3.6 \mathrm{~nm}$, calculated from the HAADF-STEM images $)$. The resistance of the glass support $\left(R_{\text {support }}=1.56 \mathrm{M} \Omega\right)$ and of the membrane on the glass support $\left(R_{\text {support }}+R_{\text {membrane }}=1.95 \mathrm{M} \Omega\right)$ are calculated from the data presented in Fig. 3b. The resistance of the $1-\mu \mathrm{m}$-wide freestanding membrane disk in $1 \mathrm{M} \mathrm{KCl}$ solution at $\mathrm{pH} 3$ is $R_{\text {membrane }}=0.39 \mathrm{M} \Omega$, thus the pore density is approximately $1.5 \times 10^{10} \mathrm{~cm}^{-2}$, which is close to the pore density obtained from the HAADF-STEM images (approximately $1.2 \times 10^{10} \mathrm{~cm}^{-2}$, obtained by analysing 33 TEM images). Besides, pores smaller than $1 \mathrm{~nm}$ in diameter cannot be identified clearly from the HAADF-STEM images, however, the ions can still translocate through those pores as determined from the $I-V$ measurements ${ }^{38-40}$. Before the transfer of the membrane, and as expected, the bare glass pore behaves as an ohmic resistor in $0.1 \mathrm{M} \mathrm{KCl}$ solution (Supplementary Fig. 12). However, after the transfer of the membrane, in contrast to the linear $I-V$ characteristics at a small bias $(\sim 40 \mathrm{mV})$, rectified $I-V$ behaviours were observed over larger bias voltages ( -200 to $200 \mathrm{mV}$ ) (Fig. 3c). The linear response of the conductance was calculated based on the data in Fig. 3c. By decreasing the concentration of the $\mathrm{KCl}$ electrolyte, the ionic conductance of the membrane over $1 \mu \mathrm{m}$ pores decreases nonlinearly, indicating the existence of charge on the membrane (Fig. 3c, inset) ${ }^{41}$.

Ion current rectification is observed for the molecularly-thin porous membranes, for a range of $\mathrm{KCl}$ concentrations and $\mathrm{pH}$ (Fig. $3 \mathrm{c}$ and Supplementary Fig. 13). At higher $\mathrm{pH}$, a stronger rectification 

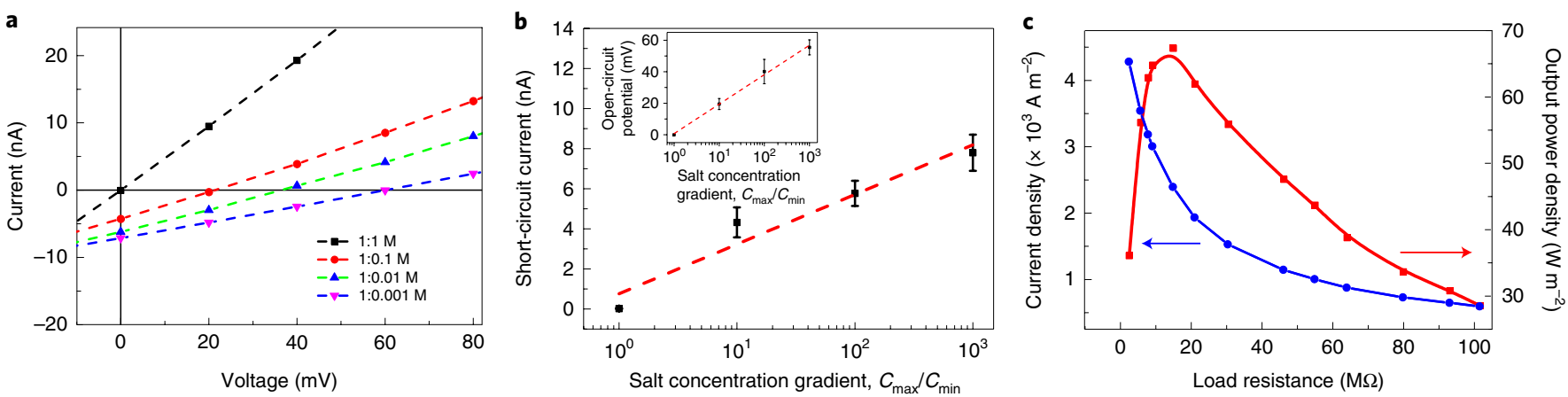

Fig. 4 | Reverse electrodialysis performance of the membrane prepared at $10 \mathrm{mN} \mathrm{m}^{-1}$. a, The $I-V$ characteristics of the membrane suspended on top of a $1 \mu \mathrm{m}$ diameter glass pore in four different $\mathrm{KCl}$ gradients at $\mathrm{pH}$ 7. $\mathbf{b}$, The generated current as a function of the salt gradient. Inset: the generated open-circuit potential as a function of the salt gradient. The error bars are standard deviations of results from three parallel experiments. c, The current density and the corresponding output power density as a function of the load resistance by mixing artificial seawater $(0.5 \mathrm{M} \mathrm{NaCl})$ and river water $(0.01 \mathrm{M} \mathrm{NaCl})$.

effect is observed, which indicates a higher surface charge. The surface charge mainly comes from the oxygen-containing groups, such as $\mathrm{COO}^{-}$. Such rectification, for example in ion channels, is usually attributed to the asymmetric charge (bipolar diodes or unipolar diodes) and/or the asymmetric geometry of the pore (for instance a conical shape $)^{42-44}$. Considering the ultrathin $(2 \mathrm{~nm})$ nature of the membrane, the rectification is more likely due to the asymmetric charge distribution as evidenced previously in chemical vapor deposition (CVD) graphene containing a single intrinsic nanopore ${ }^{38}$.

\section{Ion selectivity and electricity generation}

Furthermore, the membrane is ion selective. To demonstrate that, $\mathrm{KCl}$ solutions with concentrations ranging from $1 \mathrm{mM}$ to $1 \mathrm{M}$ were placed in the trans reservoir of the flow cell with the membrane prepared at $10 \mathrm{mN} \mathrm{m}^{-1}$ while the $1 \mathrm{M} \mathrm{KCl}$ solution was always placed in the cis reservoir. Salt bridges were used between the $\mathrm{Ag} / \mathrm{AgCl}$ electrode and the $\mathrm{KCl}$ reservoir to exclude the redox potential from the electrode in different $\mathrm{KCl}$ concentrations ${ }^{36}$. The short-circuit current $I_{s c}$ (the current corresponding to zero external bias) and opencircuit potential $E_{\text {oc }}$ (the potential corresponding to the zero current) were obtained from the $I-V$ measurements. In the absence of an applied voltage, the $I_{s c}$ is negative, indicating that the membrane is negatively charged and therefore is cation (that is, $\mathrm{K}^{+}$) selective (Fig. 4a) ${ }^{12}$. The membranes obtained at $20 \mathrm{mN} \mathrm{m}^{-1}$ and $30 \mathrm{mN} \mathrm{m}^{-1}$ (Supplementary Figs. 14 and 15) showed a similar short-circuit current and open-circuit potential.

As shown in Fig. 4b, the $I_{\mathrm{sc}}$ and $E_{\mathrm{oc}}$ increase almost linearly with the logarithm of the $\mathrm{KCl}$ concentration gradient between the two reservoirs. The ion selectivity can be calculated from $E_{\text {oc }}$ using equation $(2)^{12,45,46}$

$$
E_{\mathrm{oc}}=\left(t_{+}-t_{-}\right) \frac{R T}{z F} \ln \frac{\gamma_{\mathrm{high}} c_{\mathrm{high}}}{\gamma_{\mathrm{low}} c_{\mathrm{low}}}
$$

where $R, T, z, F, \gamma, c, t_{+}$and $t$ are the gas constant, temperature, ion valence, Faraday constant, mean activity coefficient, salt concentration and the transference number for cation and anion, respectively. The transference number $t_{+}$and $t_{-}$shows the selectivity for cations and anions, and is related to the cation and anion fluxes $\left(j_{+}\right.$and $\left.j_{-}\right)$ via the equation $t_{+}=j_{+} /\left(j_{+}+j_{-}\right)$. The ion selectivity of the membrane is defined as $S=t_{+}-t_{-}$, calculated using equation (2). The ion selectivity $S$ of the membrane is about $40 \%$, which is comparable to the results of $2 \mathrm{D}$ materials reported in the literature ${ }^{12}$.

We also demonstrate that the membrane can be used to generate electricity from mixing artificial seawater $(0.5 \mathrm{M} \mathrm{NaCl})$ and river water $(0.01 \mathrm{M} \mathrm{NaCl})$ via reverse electrodialysis. The output power of a $1-\mu \mathrm{m}$-diameter freestanding membrane can be calculated by $P=I^{2} \times R$, where $I$ is the current and $R$ is the load resistance. The current decreases as the load resistance increases and the output power reaches a maximum value of $67 \mathrm{~W} \mathrm{~m}^{-2}$ with a load resistance of $15 \mathrm{M} \Omega$ (Fig. 4c). Because the ion selectivity of the membrane reported here is lower than the commonly used reverse electrodialysis membrane, the open-circuit potential $E_{\mathrm{oc}}$ is also lower. In fact, the molecularly-thin nature of the membrane gives rise to an ionic conductivity larger than the thick commercially available membrane, and therefore a large short-circuit current $I_{\mathrm{sc}}$. Consequently, the overall maximum output power is still approximately two orders of magnitude higher than that of the classic exchange membranes and the current prototype nanoporous membranes reported in the literature (Supplementary Table 3). We also demonstrate the possibility to get a centimetre-size membrane on the porous support (a trace-etched polycarbonate membrane) without any cracks, which can be used for large-area energy generation (Supplementary Fig. 16). We envisage that the reverse electrodialysis performance could be further improved by using support materials with lower intrinsic ionic resistance, introducing a higher surface charge and higher pore density ${ }^{47}$.

In summary, we have synthesized centimetre-sized, molecularlythin nanoporous carbon-based membranes. The core-rim structure of the monomer is important for the membrane preparation: the core is thermally stable, which forms the frame of the membrane; the rim is thermally unstable, which decomposes and crosslinks the cores. Additionally, the fact that the membrane is ultra-permeable to cations makes it a good candidate for reverse electrodialysis research. By using a diverse set of core-rim monomers, our strategy paves a way towards the controllable design and synthesis of atomically thin porous membranes with a tight pore size distribution and varied frame structure, and, importantly, provides the possibility for the large-scale fabrication of nanopore arrays yielding remarkable ionic selectivity and conductivity that are crucial for water purification, desalination and proton transport.

\section{Online content}

Any methods, additional references, Nature Research reporting summaries, source data, extended data, supplementary information, acknowledgements, peer review information; details of author contributions and competing interests; and statements of data and code availability are available at https://doi.org/10.1038/s41565020-0641-5.

Received: 30 January 2018; Accepted: 13 January 2020; Published online: 9 March 2020 


\section{References}

1. Logan, B. E. \& Elimelech, M. Membrane-based processes for sustainable power generation using water. Nature 488, 313-319 (2012).

2. Schroeder, T. B. H. et al. An electric-eel-inspired soft power source from stacked hydrogels. Nature 552, 214-218 (2017).

3. Mei, Y. \& Tang, C. Y. Recent developments and future perspectives of reverse electrodialysis technology: a review. Desalination 425, 156-174 (2018).

4. Yip, N. Y., Brogioli, D., Hamelers, H. V. M. \& Nijmeijer, K. Salinity gradients for sustainable energy: primer, progress, and prospects. Environ. Sci. Technol. 50, 12072-12094 (2016).

5. Giorno, L, Drioli, E. \& Strathmann, H. Permselectivity of ion-exchange membranes in Encyclopedia of Membranes (Springer, 2016).

6. Guo, X. et al. Permselectivity and conductivity of membranes based on sulfonated naphthalenic copolyimides. J. Phys. Chem. B 111, 13694-13702 (2007)

7. Yip, N. Y. \& Elimelech, M. Comparison of energy efficiency and power density in pressure retarded osmosis and reverse electrodialysis. Environ. Sci. Technol. 48, 11002-11012 (2014).

8. Geise, G. M., Hickner, M. A. \& Logan, B. E. Ionic resistance and permselectivity tradeoffs in anion exchange membranes. ACS Appl. Mater. Interfaces 5, 10294-10301 (2013).

9. Macha, M., Marion, S., Nandigana, V. V. R. \& Radenovic, A. 2D materials as an emerging platform for nanopore-based power generation. Nat. Rev. Mater. 4, 588-605 (2019).

10. Sint, K., Wang, B. \& Král, P. Selective ion passage through functionalized graphene nanopores. J. Am. Chem. Soc. 130, 16448-16449 (2008).

11. Siria, A. et al. Giant osmotic energy conversion measured in a single transmembrane boron nitride nanotube. Nature 494, 455-458 (2013).

12. Feng, J. D. et al. Single-layer $\mathrm{MoS}_{2}$ nanopores as nanopower generators. Nature 536, 197-200 (2016).

13. Yang, Y. et al. Large-area graphene-nanomesh/carbon-nanotube hybrid membranes for ionic and molecular nanofiltration. Science 364, 1057-1062 (2019).

14. Moreno, C. et al. Bottom-up synthesis of multifunctional nanoporous graphene. Science 360, 199-203 (2018).

15. Angelova, P. et al. A universal scheme to convert aromatic molecular monolayers into functional carbon nanomembranes. ACS Nano 7 6489-6497 (2013).

16. Bauer, T. et al. Synthesis of free-standing, monolayered organometallic sheets at the air/water interface. Angew. Chem. Int. Ed. 50, 7879-7884 (2011).

17. Zheng, Z. et al. Synthesis of two-dimensional analogues of copolymers by site-to-site transmetalation of organometallic monolayer sheets. J. Am. Chem. Soc. 136, 6103-6110 (2014).

18. Nishikata, Y., Konishi, T., Morikawa, A., Kakimoto, M.-a. \& Imai, Y. Preparation and monolayer thickness of Langmuir-Blodgett films of polyimides having various chemical structures. Polym. J. 20, 269-272 (1988).

19. Silva, N. J., Machado, F. B. C., Lischka, H. \& Aquino, A. J. A. $\pi-\pi$ stacking between polyaromatic hydrocarbon sheets beyond dispersion interactions. Phys. Chem. Chem. Phys. 18, 22300-22310 (2016).

20. Schneider, G. F., Calado, V. E., Zandbergen, H., Vandersypen, L. M. K. \& Dekker, C. Wedging transfer of nanostructures. Nano Lett. 10, 1912-1916 (2010).

21. Bilkan, M. T., Şahin, O. \& Yurdakul, Ş. Experimental and DFT studies of solvent effects on molecular structure and physical properties of dipyridylamine pyridine based ligand. J. Mol. Struct. 1133, 580-590 (2017).

22. Anariba, F., Viswanathan, U., Bocian, D. F. \& McCreery, R. L. Determination of the structure and orientation of organic molecules tethered to flat graphitic carbon by ATR-FT-IR and Raman spectroscopy. Anal. Chem. 78, 3104-3112 (2006)

23. Maghsoumi, A. et al. Edge chlorination of hexa-peri-hexabenzocoronene investigated by density functional theory and vibrational spectroscopy. Phys. Chem. Chem. Phys. 18, 11869-11878 (2016).

24. Beloded, A. A., Koshechko, V. G., Pokhodenko, V. D., Nemoshkalenko, V. V. \& Aleshin, V. G. X-ray photoelectron spectra of radical cations of the triphenylamine series and some heterocyclic compounds. Theor. Exp. Chem. 17, 99-103 (1981).
25. Susi, T., Pichler, T. \& Ayala, P. X-ray photoelectron spectroscopy of graphitic carbon nanomaterials doped with heteroatoms. Beilstein J. Nanotechnol. 6, 177-192 (2015).

26. Yuan, K. et al. 2D heterostructures derived from $\mathrm{MoS}_{2}$-templated, cobaltcontaining conjugated microporous polymer sandwiches for the oxygen reduction reaction and electrochemical energy storage. ChemElectroChem 4, 709-715 (2017)

27. Johra, F. T., Lee, J.-W. \& Jung, W.-G. Facile and safe graphene preparation on solution based platform. J. Ind. Eng. Chem. 20, 2883-2887 (2014).

28. Wu, Z.-S. et al. Bottom-up fabrication of sulfur-doped graphene films derived from sulfur-annulated nanographene for ultrahigh volumetric capacitance micro-supercapacitors. J. Am. Chem. Soc. 139, 4506-4512 (2017).

29. Ma, Z. et al. Sulfur-doped graphene derived from cycled lithium-sulfur batteries as a metal-free electrocatalyst for the oxygen reduction reaction. Angew. Chem. Int. Ed. 54, 1888-1892 (2015).

30. Zuo, C. \& Jagodzinski, P. W. Surface-enhanced Raman scattering of pyridine using different metals: differences and explanation based on the selective formation of $\alpha$-pyridyl on metal surfaces. J. Phys. Chem. B 109, 1788-1793 (2005)

31. Matsuoka, R. et al. Crystalline graphdiyne nanosheets produced at a gas/ liquid or liquid/liquid interface. J. Am. Chem. Soc. 139, 3145-3152 (2017).

32. Maghsoumi, A., Brambilla, L., Castiglioni, C., Müllen, K. \& Tommasini, M. Overtone and combination features of $\mathrm{G}$ and $\mathrm{D}$ peaks in resonance Raman spectroscopy of the $\mathrm{C}_{78} \mathrm{H}_{26}$ polycyclic aromatic hydrocarbon. J. Raman Spectrosc. 46, 757-764 (2015).

33. Verzhbitskiy, I. A. et al. Raman fingerprints of atomically precise graphene nanoribbons. Nano Lett. 16, 3442-3447 (2016).

34. Mao, J., Cao, X., Olk, D. C., Chu, W. \& Schmidt-Rohr, K. Advanced solid-state NMR spectroscopy of natural organic matter. Prog. Nucl. Magn. Reson. Spectrosc. 100, 17-51 (2017).

35. Wang, S., Tang, Y., Schobert, H. H., Guo, Yn \& Su, Y. FTIR and ${ }^{13} \mathrm{C}$ NMR investigation of coal component of Late Permian coals from Southern China. Energy Fuels 25, 5672-5677 (2011).

36. Rollings, R. C., Kuan, A. T. \& Golovchenko, J. A. Ion selectivity of graphene nanopores. Nat. Commun. 7, 11408 (2016).

37. Wang, L. et al. Fundamental transport mechanisms, fabrication and potential applications of nanoporous atomically thin membranes. Nat. Nanotechnol. 12 509-522 (2017).

38. Jain, T. et al. Heterogeneous sub-continuum ionic transport in statistically isolated graphene nanopores. Nat. Nanotechnol. 10, 1053-1057 (2015).

39. Feng, J. D. et al. Observation of ionic Coulomb blockade in nanopores. Nat. Mater. 15, 850-855 (2016).

40. Esfandiar, A. et al. Size effect in ion transport through angstrom-scale slits. Science 358, 511-513 (2017).

41. Gao, J. et al. High-performance ionic diode membrane for salinity gradient power generation. J. Am. Chem. Soc. 136, 12265-12272 (2014).

42. Plett, T. S. et al. Solid-state ionic diodes demonstrated in conical nanopores. J. Phys. Chem. C 121, 6170-6176 (2017).

43. Siwy, Z. S. \& Howorka, S. Engineered voltage-responsive nanopores. Chem. Soc. Rev. 39, 1115-1132 (2010).

44. Karnik, R., Duan, C. H., Castelino, K., Daiguji, H. \& Majumdar, A. Rectification of ionic current in a nanofluidic diode. Nano Lett. 7 , 547-551 (2007)

45. Ouyang, W., Wang, W., Zhang, H. X., Wu, W. G. \& Li, Z. H. Nanofluidic crystal: a facile, high-efficiency and high-power-density scaling up scheme for energy harvesting based on nanofluidic reverse electrodialysis. Nanotechnology 24, 345401 (2013).

46. Weinstein, J. N. \& Leitz, F. B. Electric power from differences in salinity: the dialytic battery. Science 191, 557-559 (1976).

47. Cao, L. et al. On the origin of ion selectivity in ultrathin nanopores: insights for membrane-scale osmotic energy conversion. Adv. Funct. Mater. 28, 1804189 (2018).

Publisher's note Springer Nature remains neutral with regard to jurisdictional claims in published maps and institutional affiliations.

(C) The Author(s), under exclusive licence to Springer Nature Limited 2020 


\section{Methods}

The chemicals are commercially available and were used without further purification. Hexa( $p$-bromophenyl)benzene and hexabromohexabenzocoronene were synthesized following a previously reported procedure ${ }^{48,49}$. A glass disc (NPC-1 Nanion) with a 1- $\mu \mathrm{m}$-wide through-hole was purchased from Nanion 228 Technologies. NMR spectra were measured on a Bruker DPX-300 spectrometer. High resolution mass spectrometry (HRMS) was recorded by a Thermo Scientific LTQ Orbitrap XL high-resolution Fourier-transform mass spectrometry (FT-MS) system with electrospray ionization source (ESI). FTIR spectra were recorded on a Perkin-Elmer Paragon 1000 FTIR spectrophotometer equipped with a Golden Gate attenuated total reflection (ATR) device. Optical images were recorded on a Leica DM $2700 \mathrm{M}$ microscope. AFM images were recorded on a JPK Nano Wizard Ultra Speed machine with a silicon 254 probe (AC 160 TS, Asylum Research) with $300 \mathrm{kHz}$ nominal resonance frequency. The images were scanned in intermittent contact mode in air at room temperature. The SEM images were recorded by using an FEI NOVA nano SEM 200 scanning electron microscope. The membrane was transferred from a copper substrate directly onto a gold TEM grid with carbon mesh (QUANTIFOIL, Au 200 mesh) following standard procedure ${ }^{50}$. Raman spectra of the monomer HPAHBC and membranes were recorded on a WITec confocal spectrometer with a $532 \mathrm{~nm}$ laser and a 100× objective. The TEM experiment was conducted on an image-side Cs-corrected FEI Titan 80-300 microscope operated at $300 \mathrm{kV}$. All the magic-angle spinning (MAS) solidstate NMR (SSNMR) measurements were taken with a Bruker AV-I $750 \mathrm{MHz}$ spectrometer with a $17.6 \mathrm{~T}$ magnetic field. In this field ${ }^{13} \mathrm{C}$ and ${ }^{1} \mathrm{H}$ resonate at 188.66 and $750.23 \mathrm{MHz}$, respectively. A standard $4 \mathrm{~mm}$ triple resonance MAS probe was used. All the samples were packed in $4 \mathrm{~mm}$ zirconium rotors and were spun at the magic angle (54.74) at various spinning speeds. The ${ }^{13} \mathrm{C}$ spectrum was obtained through the CPMAS ${ }^{51,52}$ technique with SPINAL- $64^{53}$ decoupling. CPMAS is optimized by irradiating ${ }^{1} \mathrm{H}$ and ${ }^{13} \mathrm{C}$ with $80.6 \mathrm{kHz}$ and $40.32 \mathrm{kHz}$ radio-frequency pulses for a contact time of $2 \mathrm{~ms}$. A recycle delay of $1 \mathrm{~s}$ and 10,240 scans were acquired. The line broadening function of $200 \mathrm{~Hz}$ was applied while processing the spectra. All the ${ }^{13} \mathrm{C}$ spectra were externally referenced to the methyl signal of tetramethylsilane (TMS) and were processed in Topspin 3.2 and MestReNova software. Nanofluidic measurements were carried out using a low noise patchclamp set-up with a $100 \mathrm{kHz}$ bandwidth amplifier (Axopatch 200B, Axon Instruments) equipped with two $\mathrm{Ag} / \mathrm{AgCl}$ electrodes connected, respectively, to the anode and cathode of the amplifier. The home-made salt bridge was prepared following a previously reported procedure $e^{54,55}$.

Synthesis of monomer HPAHBC. A mixture of dipyridylamine $(0.34 \mathrm{~g}, 2.0 \mathrm{mmol})$, hexabromohexabenzocoronene $(0.10 \mathrm{~g}, 0.1 \mathrm{mmol}), \mathrm{K}_{2} \mathrm{CO}_{3}(0.14 \mathrm{~g}, 1.0 \mathrm{mmol})$ and $\mathrm{CuSO}_{4}(0.01 \mathrm{~g}, 0.06 \mathrm{mmol})$ was placed in a $10 \mathrm{ml}$ flask and heated at $190^{\circ} \mathrm{C}$ under $\mathrm{N}_{2}$ for 4 days. After the reaction, the black solid was first dissolved in dichloromethane (DCM) and filtered. The filtrate was concentrated in vacuum, and purified by chromatography with DCM as eluent. The final product was a yellowish solid with about $10 \%$ penta $(2,2$ '-dipyridylamino)hexabenzocoronene (0.04 g, yield 26\%) (Supplementary Figs. $17-23) .{ }^{1} \mathrm{H}$ NMR $\left(\mathrm{CDCl}_{3}, 300 \mathrm{MHz}\right)$ : $\delta=8.71(\mathrm{~s}, 12 \mathrm{H}), 8.35(\mathrm{~d}, J=4.0 \mathrm{~Hz}, 12 \mathrm{H}), 7.63-7.54(\mathrm{~m}, 12 \mathrm{H}), 7.14(\mathrm{~d}, J=8.3 \mathrm{~Hz}$, $12 \mathrm{H}), 7.02-6.95(\mathrm{~m}, 12 \mathrm{H}) .{ }^{13} \mathrm{C} \mathrm{NMR}\left(\mathrm{CDCl}_{3}, 75 \mathrm{MHz}\right): \delta=158.2,148.7,143.9$, $137.9,131.9,123.7,121.6,120.8,118.6,117.3 \mathrm{ppm}$. IR $(v): 3,054(\mathrm{w}), 3,003(\mathrm{w})$, $2,925(\mathrm{w}), 2,853(\mathrm{w}), 1,585(\mathrm{~s}), 1,468(\mathrm{~s}), 1,429$ (vs), 1,375 (m), 1,329 (m), 1,260 (m), 1,152 (w), $773(\mathrm{w}), 742(\mathrm{w}) \mathrm{cm}^{-1}$. HRMS (ESI) found (calc): 769.2732 (769.2703) $[1 / 2(\mathrm{M}+2 \mathrm{H})]^{+}$.

Membrane fabrication. The membrane was prepared by a two-step method. The Langmuir-Blodgett step was done with a Langmuir-Blodgett trough (KSV NIMA, Finland). The surface pressure was measured with a Wilhelmy balance. A HPAHBC/CHCl ${ }_{3}$ solution $\left(35 \mu \mathrm{l}, 1 \mathrm{mg} \mathrm{ml}^{-1}\right)$ was spread at the interface of water and air carefully. After $30 \mathrm{~min}$, the HPAHBC was compressed by the barrier with a speed of $2 \mathrm{~mm} \mathrm{~min}^{-1}$ to reach a certain surface pressure $\left(3 \mathrm{mN} \mathrm{m}^{-1}, 10 \mathrm{mN} \mathrm{m}^{-1}\right.$, $20 \mathrm{mN} \mathrm{m}^{-1}$ and $30 \mathrm{mN} \mathrm{m}^{-1}$ ). The HPAHBC layer was then transferred onto a copper substrate by the Langmuir-Schäfer method. The copper substrate with the HPAHBC layer was delivered into a CVD oven, and thermally annealed under vacuum ( $1 \mathrm{mbar}$, argon atmosphere) for $20 \mathrm{~min}$ at $550^{\circ} \mathrm{C}$. Then the CVD oven was cooled down, and the membrane on the copper substrate was taken out and used for further measurements. The membrane could not be obtained without a thermal treatment or with thermal annealing below $550^{\circ} \mathrm{C}$ (Supplementary Fig. 24). The copper substrate plays the role of a support rather than a catalyst in the membrane formation and can be replaced by other materials, such as gold (Supplementary Fig. 25).

Simulations. Geometry optimization of the HPAHBC molecule in the gas phase was performed using density functional theory (DFT) with the BLYP-D3 functional and TZP basis set using the ADF2018 program ${ }^{56}$. The cartesian coordinates of the optimized geometry are given in Supplementary Table $4 .{ }^{13} \mathrm{C}$ chemical shifts (ppm) were consistently computed at DFT level with the BLYP functional and TZP basis set using ADF2018 (Supplementary Table 2).

All MD simulations were carried out using GROMACS 2016 $6^{57-63}$ and Visual Molecular Dynamics (VMD) ${ }^{64}$ was used for visualization. The particle mesh Ewald
(PME) method was employed to accurately compute electrostatic interactions ${ }^{65}$. The cut-off for the Coulomb interaction and Lennard-Jones interaction was set at $10 \AA$. During the NVT simulation, the temperature was kept fixed with the $\mathrm{V}$-rescale coupling method ${ }^{66}$.

Simulation settings for the water interfaces. A simulation of a water slab containing 4,139 water molecules in a periodic box $\left(5.0 \times 5.0 \times 20.0 \mathrm{~nm}^{3}\right)$ has been carried out to establish a water model using the TIP4P-Ew/2004 force field ${ }^{67}$. The system was first energetically minimized and then was equilibrated for $8 \mathrm{~ns}$ with NVT at $300 \mathrm{~K}$ to obtain an average surface tension of $\gamma_{\text {water }}=58.46 \mathrm{mN} \mathrm{m}^{-1}$. This value is in good agreement with previous studies ${ }^{68,69}$. Furthermore, a radial distribution function and density analysis were performed to validate our water slab (Supplementary Fig. 26).

Simulation settings for the water/HPAHBC interfaces. In the MD simulation of HPAHBC molecules on water, the organic molecules were simulated using the OPLS-AA force field (Supplementary Table 4) ${ }^{70-72}$. We packed a specific amount of previously optimized (at DFT level) HPAHBC molecules in our water box using the PACKMOL18 program ${ }^{73,74}$. An equal number of HPAHBC molecules were placed on both sides of the water slab producing two HPAHBC/water interfaces. We could improve the statistics on our results by averaging on both interfaces, which can be considered independent due to the thickness of the water box and the vacuum space above each interface along the $z$ axis.

We initialized the simulations with a different number of HPAHBC molecules, from 1 to 6 , on each side of the slab. For each case the molecules were initially arranged in three different starting configurations, vertical, stacked and planar (Extended Data Fig. 1a-c). Each system was first equilibrated with NVT at $70 \mathrm{~K}$ for $2.5 \mathrm{~ns}$, and then the temperature was increased to $300 \mathrm{~K}$ for the other $2.5 \mathrm{~ns}$. After these pre-equilibration simulations, we ran a final NVT simulation at $300 \mathrm{~K}$ for $10 \mathrm{~ns}$, which was used for our data production and analysis ${ }^{75}$.

\section{Data availability}

Source data for Figs. 1-4 and Extended Data Fig. 1 are provided with the paper All other data that support the plots within this paper and other findings of this study are available from the corresponding author on reasonable request.

\section{References}

48. Jia, W. L. et al. 7-azaindolyl- and 2,2'-dipyridylamino-functionalized molecular stars with sixfold symmetry: self-assembly, luminescence, and coordination compounds. Chem. Eur. J. 11, 832-842 (2005).

49. Thompson, C. M. et al. Substituent effects on the gas sorption and selectivity properties of hexaphenylbenzene and hexabenzocoronene based porous polymers. Macromolecules 47, 8645-8652 (2014).

50. Pantelic, R. S. et al. Graphene: substrate preparation and introduction. J. Struct. Biol. 174, 234-238 (2011).

51. Hartmann, S. R. \& Hahn, E. L. Nuclear double resonance in the rotating frame. Phys. Rev. 128, 2042-2053 (1962).

52. Metz, G., Wu, X. L. \& Smith, S. O. Ramped-amplitude cross polarization in magic-angle-spinning NMR. J. Magn. Reson. Ser. A 110, 219-227 (1994).

53. Fung, B. M., Khitrin, A. K. \& Ermolaev, K. An improved broadband decoupling sequence for liquid crystals and solids. J. Magn. Reson. 142, 97-101 (2000)

54. Martín-Yerga, D., Rama, E. C. \& Costa-García, A. Electrochemical study and applications of selective electrodeposition of silver on quantum dots. Anal. Chem. 88, 3739-3746 (2016).

55. Barlag, R. et al. A student-made silver-silver chloride reference electrode for the general chemistry laboratory: $\sim 10 \mathrm{~min}$ preparation. J. Chem. Educ. 91, 766-768 (2014).

56. Baerends, E. J. et al. ADF2017, SCM, Theoretical Chemistry https://www.scm. com (Vrije Universiteit, Amsterdam, The Netherlands).

57. Berendsen, H. J. C., van der Spoel, D. \& van Drunen, R. GROMACS: a message-passing parallel molecular dynamics implementation. Comput. Phys. Commun. 91, 43-56 (1995).

58. Lindahl, E., Hess, B. \& van der Spoel, D. GROMACS 3.0: a package for molecular simulation and trajectory analysis. J. Mol. Model. 7 , 306-317 (2001).

59. Van Der Spoel, D. et al. GROMACS: fast, flexible, and free. J. Comput. Chem. 26, 1701-1718 (2005).

60. Hess, B., Kutzner, C., van der Spoel, D. \& Lindahl, E. GROMACS 4: algorithms for highly efficient, load-balanced, and scalable molecular simulation. J. Chem. Theory Comput. 4, 435-447 (2008).

61. Pronk, S. et al. GROMACS 4.5: a high-throughput and highly parallel open source molecular simulation toolkit. Bioinformatics 29, 845-854 (2013).

62. Abraham, M. J. et al. GROMACS: high performance molecular simulations through multi-level parallelism from laptops to supercomputers. SoftwareX 1-2, 19-25 (2015).

63. Páll, S., Abraham, M. J., Kutzner, C., Hess, B. \& Lindahl, E. in Solving Software Challenges for Exascale (eds Markidis, S. \& Laure, E.) 3-27 (Springer International Publishing, 2015). 
64. Humphrey, W., Dalke, A. \& Schulten, K. VMD: visual molecular dynamics. J. Mol. Graph. 14, 33-38 (1996).

65. York, D. M., Darden, T. A. \& Pedersen, L. G. The effect of long-range electrostatic interactions in simulations of macromolecular crystals: a comparison of the Ewald and truncated list methods. J. Chem. Phys. 99, 8345-8348 (1993).

66. Bussi, G., Donadio, D. \& Parrinello, M. Canonical sampling through velocity rescaling. J. Chem. Phys. 126, 014101 (2007).

67. Horn, H. W. et al. Development of an improved four-site water model for biomolecular simulations: TIP4P-Ew. J. Chem. Phys. 120, 9665-9678 (2004)

68. Vega, C. \& de Miguel, E. Surface tension of the most popular models of water by using the test-area simulation method. J. Chem. Phys. 126, 154707 (2007)

69. Chen, F. \& Smith, P. E. Simulated surface tensions of common water models. J. Chem. Phys. 126, 221101 (2007).

70. Jorgensen, W. L., Maxwell, D. S. \& Tirado-Rives, J. Development and testing of the OPLS all-atom force field on conformational energetics and properties of organic liquids. J. Am. Chem. Soc. 118, 11225-11236 (1996).

71. Kaminski, G. A., Friesner, R. A., Tirado-Rives, J. \& Jorgensen, W. L. Evaluation and reparametrization of the OPLS-AA force field for proteins via comparison with accurate quantum chemical calculations on peptides. J. Phys. Chem. B 105, 6474-6487 (2001).

72. Martin, M. G. Comparison of the AMBER, CHARMM, COMPASS, GROMOS OPLS, TraPPE and UFF force fields for prediction of vapor-liquid coexistence curves and liquid densities. Fluid Phase Equilib. 248, 50-55 (2006).

73. Martínez, J. M. \& Martínez, L. Packing optimization for automated generation of complex system's initial configurations for molecular dynamics and docking. J. Comput. Chem. 24, 819-825 (2003).

74. Martínez, L., Andrade, R., Birgin, E. G. \& Martínez, J. M. PACKMOL: a package for building initial configurations for molecular dynamics simulations. J. Comput. Chem. 30, 2157-2164 (2009).

75. Roe, D. R. \& Cheatham, T. E. PTRAJ and CPPTRAJ: software for processing and analysis of molecular dynamics trajectory data. J. Chem. Theory Comput. $\mathbf{9}$, 3084-3095 (2013).

\section{Acknowledgements}

This work is supported by the Netherlands Organization for Scientific Research (Vidi 723.013.007), the European Research Council (ERC) Proof of Concept NANOPORE (no. 780004). We acknowledge Nederlandse Organisatie voor Wetenschappelijk Onderzoek (NWO) Exact and Natural Sciences for the use of the supercomputer facilities at SURFsara. H.Q. and U.K. gratefully acknowledge the financial support by the German Research Foundation (DFG) and the Ministry of Science, Research and the Arts (MWK) of Baden-Wuerttemberg in the framework of the "SALVE" (Sub-Angstrom Low-Voltage Electron Microscopy) project (DFG KA 1295/21-1). S. G. Lemay is acknowledged for the general discussion about nanofluidics. W. Fu and X. Zhang are acknowledged for the discussions about ionic conductance. J. van Gerwen and C. van Helvoirt are acknowledged for the XPS measurements.

\section{Author contributions}

G.F.S. supervised the project. X.L. performed the monomer synthesis and characterization. X.L. and M.H. performed the membrane synthesis, characterization and ionic conductance measurements. H.Q. and U.K. conducted HRTEM and HAADF-STEM measurements. D.C., G.J.A.S. and F.B. performed the MD simulations. K.B.S.S.G., D.C., G.J.A.S., F.B. and H.d.G. performed the solid-state NMR studies. All authors contributed to discussions. X.L. wrote the manuscript with help from all authors.

\section{Competing interests}

The authors declare no competing interests.

\section{Additional information}

Extended data is available for this paper at https://doi.org/10.1038/s41565-020-0641-5. Supplementary information is available for this paper at https://doi.org/10.1038/ s41565-020-0641-5.

Correspondence and requests for materials should be addressed to G.F.S.

Peer review information Nature Nanotechnology thanks Yongsheng Chen and the other, anonymous, reviewer(s) for their contribution to the peer review of this work.

Reprints and permissions information is available at www.nature.com/reprints. 
Vertical

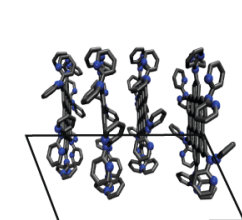

b Stacked

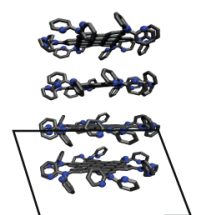

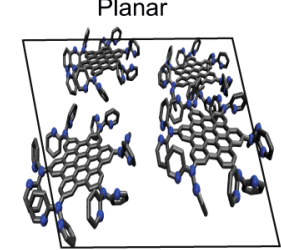

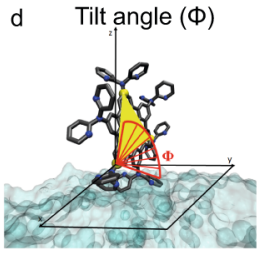

e
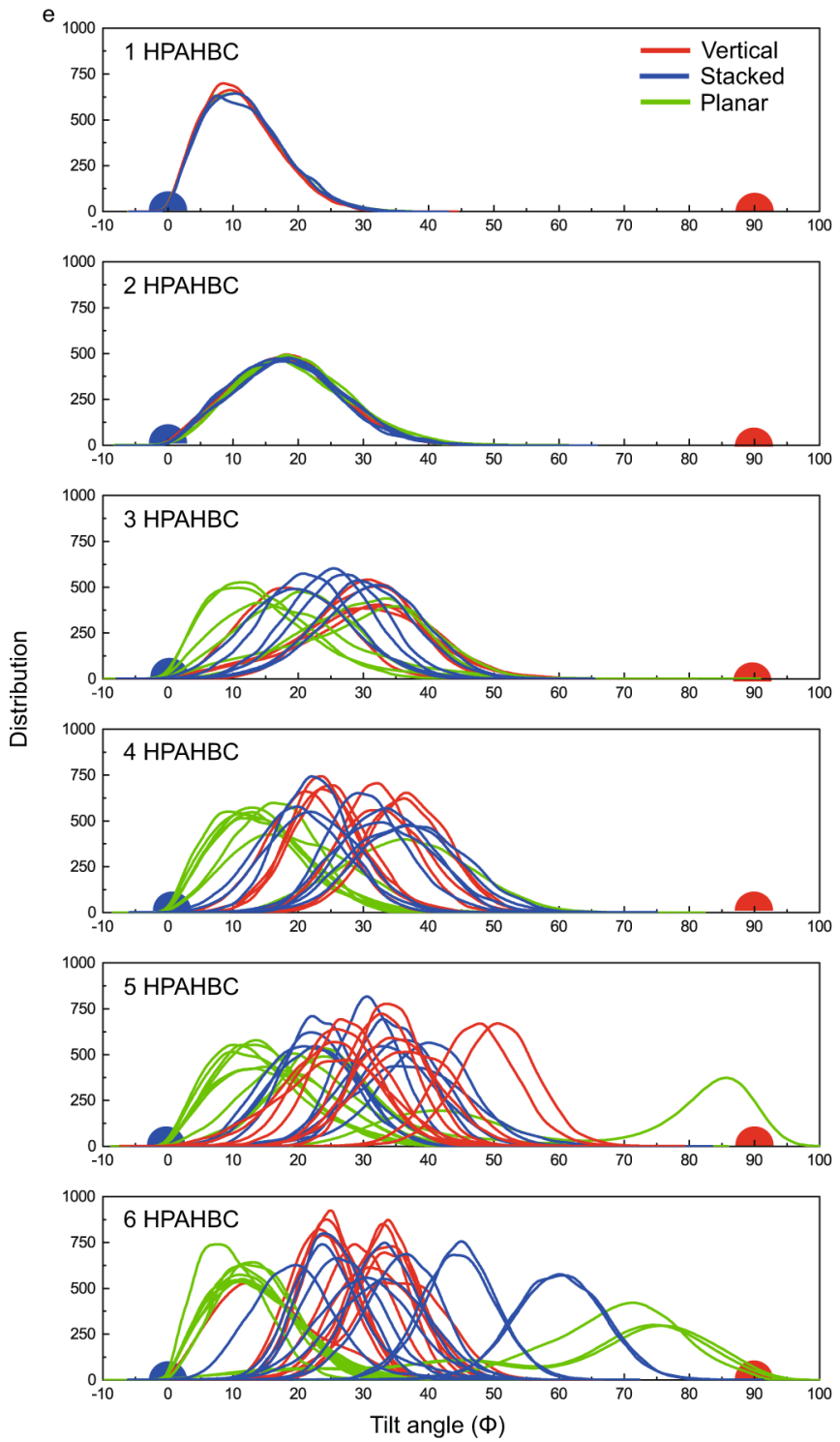

f
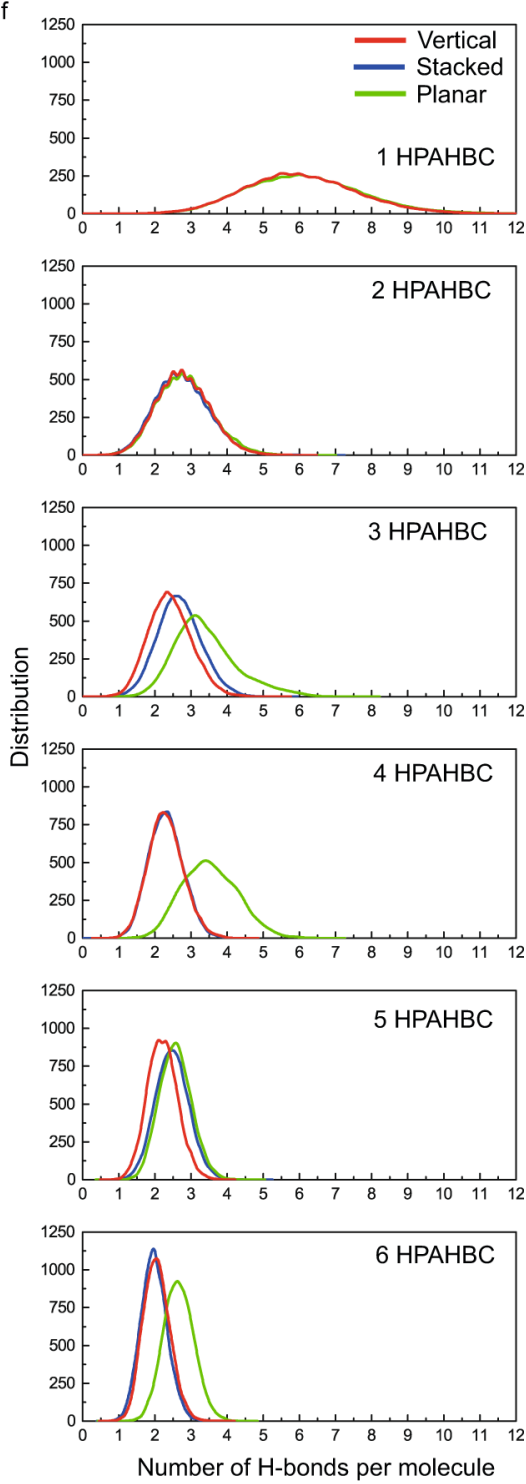

Extended Data Fig. 1 | MD simulations analysis of HPAHBC molecules on water surface. a-c, Schematic visualization of starting conformations for the HPAHBC molecules at the beginning of the MD simulation, vertical (a), stacked (b), planar (c); carbon atoms are shown in grey and nitrogen atoms in blue balls (hydrogens are omitted). d, Illustration of the tilt angle $\Phi$ (red arc) between the plane of the HBC ring defined by three specific carbon atom on the edge (yellow balls) and the $x-y$ plane; carbon atoms in grey, nitrogen atoms in blue (hydrogens are omitted). The cpptraj module of AmberTools are used for the calculation of the HBC plane. e, Distribution of tilt angle $(\Phi)$ per each molecule from MD simulations with the increasing amount of HPAHBC molecules, from 1 to 6 , and different starting point conformation, vertical (red), stacked (blue), planar (green). With the red circle at $90^{\circ}$ and the concentric blue-green circle at $0^{\circ}$, we underline the starting point for the tilt angle at the beginning of the pre-equilibration simulations, for vertical and stacked-planar conformation, respectively. f, Distribution of number of hydrogen bonds (H-bonds) per HPAHBC molecule from MD simulations with increasing amount of the HPAHBC molecules, from 1 to 6, and different starting point conformation, vertical (red), stacked (blue), planar (green).

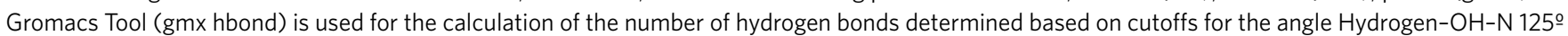
and the distance $\mathrm{OH}-\mathrm{N} 3.5 \AA$. OH is regarded as the donor, N as the acceptor. The distribution curves were obtained via Gaussian broadening with default standard deviation. 


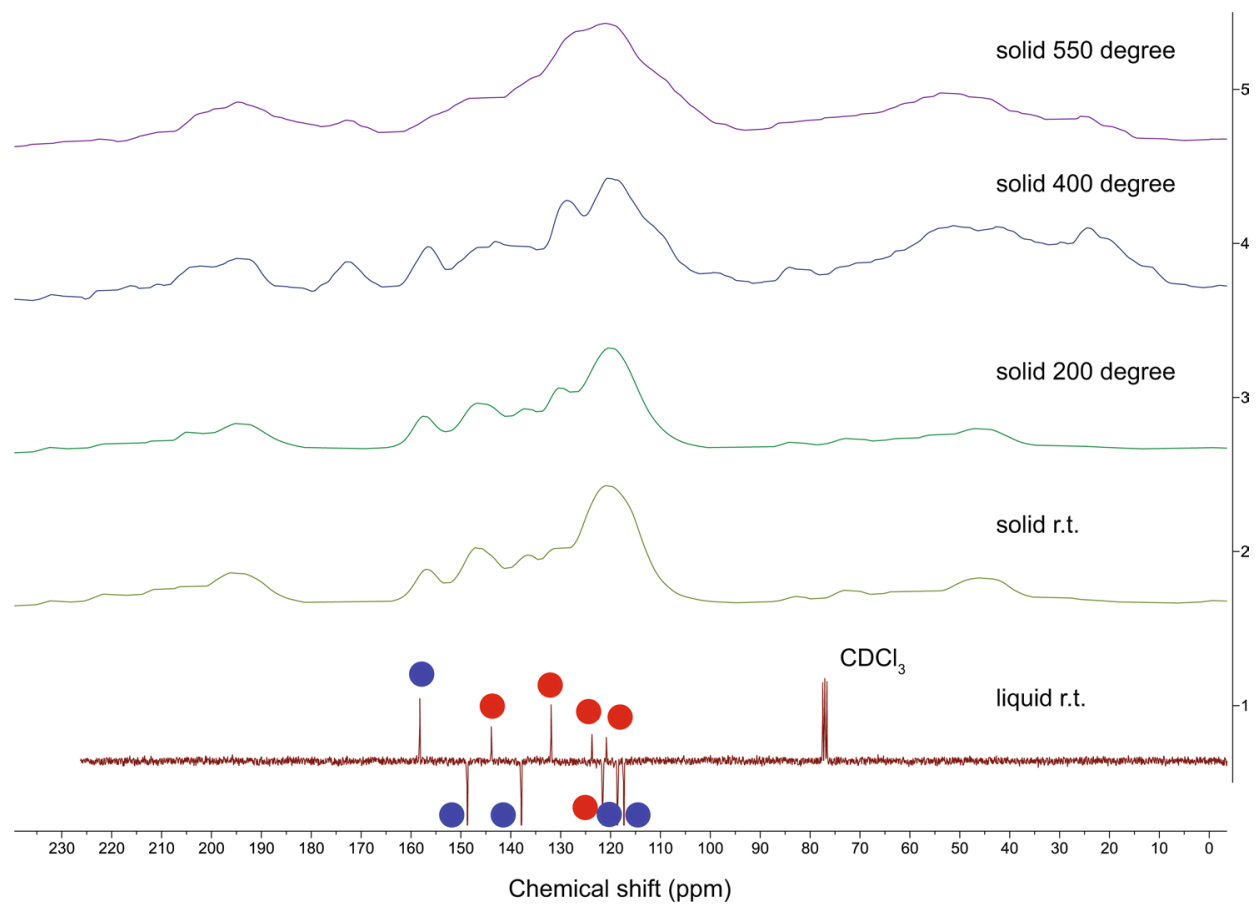

Extended Data Fig. 2 | Solid-state NMR spectra of HPAHBC powder annealed at different temperatures. As the temperature increases from 400 degrees to 550 degrees, the peaks marked with blue dots gradually vanish, which is attributed to decomposition of the dipyridylamino rim. 